\title{
Detection of Protein Molecules by Electrical Current Response Using Two-Electrode Method
}

\author{
Hong-Kun Lyu', Sung-Ho Woo ${ }^{1}$, Yoon Soo Han², Hee-Ho Lee ${ }^{3}$, and Jang-Kyoo Shin ${ }^{3,+}$
}

\begin{abstract}
In order to protect human lives from disease, various biosensors having the potential to analyze a variety of biomolecules have been utilized. Biosensors constitute one of the most promising ways to monitor and detect various biomolecules corresponding to diseases. In this study, we demonstrate that the reaction of streptavidin molecules with biotin on a gold electrode can be detected using the twoelectrode method with a gold electrode and a platinum reference electrode. We also show the characteristics of the electrical current response. While detecting $2-\mu \mathrm{M}$ streptavidin molecules dissolved in phosphate buffered saline(PBS) solution, we found that an analytical biosensor can operate on the principle of detecting an antigen-antibody reaction event of protein molecules using the two-electrode method. We think that the "potential step" method might be useful to detect the occurrence of any antigen-antibody reactions and can be combined with other devices or ICs such as BJTs, MOSFETs, and OP-amps for the detection of biomolecules of diseases.
\end{abstract}

Keywords : Biomolecules, Biosensors, Current response

\section{INTRODUCTION}

There has been a recent increase in the instances of humans being affected by pathogens such as viruses, bacteria and so on. The monitoring of pathogens is very important for the prevention of nosocomial infections. Pathogens are also biomolecules, so various biosensors have been utilized to detect different varieties of biomolecules for the maintenance and protection of general public health. These biosensors are referred to as immunosensors, which are devices comprising an antigen or antibody species coupled to a signal transducer, which detects the binding of the complementary species[1]. They can be used in the medical and food industries, as well as in the detection of toxic substances in the environment; so, there is a continuous demand for research and development into biosensors[2, 3].

Amperometric immunosensors are one of the devices

\footnotetext{
${ }^{1}$ Green Energy Research Division, Daegu-Gyeongbuk Institute of Science and Technology(DGIST), Daegu, 711-873, Republic of Korea

2 Department of Advanced Energy Material Science and Engineering, Catholic University of Daegu, Gyeongbuk, 712-702, Republic of Korea

${ }^{3}$ School of Electrical Engineering and Computer Science, Kyungpook

National University, Daegu 702-701, Republic of Korea

${ }^{+}$Corresponding author : jkshin@ee.knu.ac.kr

(Received : Dec. 30, 2010, Revised : Mar. 9, 2011,

Accepted : Mar. 16, 2011)
}

that have been used, because these sensor systems have several advantages, such as their low cost, mass producibility, and miniaturization. The amperometry method, which is used for amperometric immunosensors, is a dynamic process that measures the current or electron flow through electrodes $[4,5]$. Generally, this refers to the control of oxidation-reduction reactions on the surface of the electrode. The three-electrode method is typically used for experiments with electrochemical immunosensors. These three electrodes are the working electrode on which the biological reaction occurs, the reference electrode that behaves as the "basis potential" level, and it is comparable to the "applied potential" of the working electrode, and the counter electrode, toward which the current flows starting from the working electrode[6, 7]. In this paper, we propose that it is possible to detect an antigen-antibody reaction event of protein molecules using the two-electrode method and potential step method. Since the two electrode system is simpler than the three electrode system and can be used under conditions where the IR-drop is small, we use this method[7]. This also enables a cheaper sensor strip and simpler measurement system to be developed, because these sensor electrodes and their measurement circuits can be readily adopted for the sensor system. 


\section{EXPERIMENT}

\subsection{Principle}

An electrode in which electron transfer cannot occur across the electrode-electrolyte interface, regardless of the potential imposed by an external supply potential, is called a polarized electrode. An electrical double layer is formed at the electrode-electrolyte interface by the contact of the polarized electrode to some electrolyte solution. Negative and positive layers are formed at the interfaces of the electrode and electrolyte solution, respectively. In this study, oxidation means a chemical reaction in which an atom or ion loses electrons and the atomic number or proton number therefore increases. Reduction or deoxidation means that an atom or ion gains electrons and therefore the atomic number decreases. When a potential, which is sufficient to generate a reduction current, is applied to the electrode, a large reduction in current occurs across the electrode-electrolyte interface, and it decreases with time[4].

Fig. 1(a) and (b) show a schematic diagram of the experimental setup using the "potential step" method and the waveform used for the experiment, respectively. Fig. 1(c) shows the current response as a function of time, indicating that a very large current is generated in an instant and that it subsequently decreases exponentially, because a lot of positive ions are deoxidized around the electrode-electrolyte interface. Generally, it has been reported that the positive ions existing or arriving at the electrode-electrolyte interface are deoxidized immediately, because the reaction of oxidization and reduction is very fast[8]. Therefore, all of the positive ions disappear at the interface of the electrolyte. However, immediately thereafter, the diffusion of the positive ions occurs from the bulk solution near the interface to the electrode-electrolyte interface, and the mass transfer of chemical species including positive ions is generated in the solution. Then, the positive ions transferred from the bulk solution are deoxidized again, so that a reduction current occurs again. Over time, the diffusion of the positive ions throughout the solution occurs, owing to the differences in concentration of the positive ions.

Considering that some charged molecule layers are immobilized on the surface of the electrode, from the impedance point of view, the molecular layer hinders the electron transfer from the electrode to the electrolyte solution and the current flow is therefore decreased[5, 6].
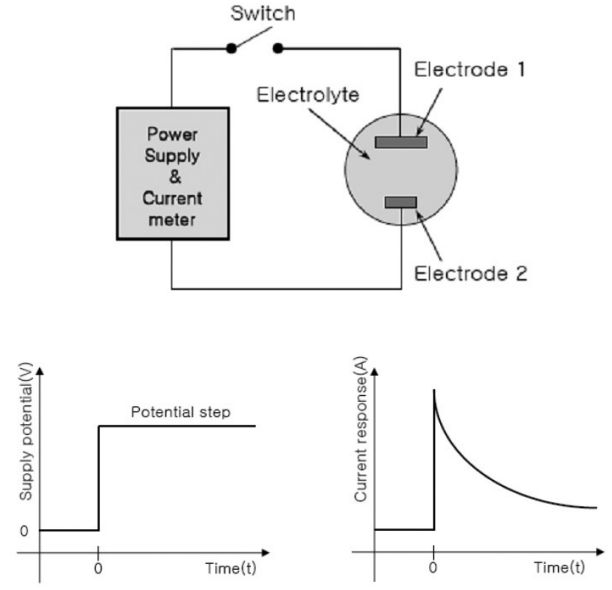

Fig. 1. Schematic diagram for the experiment using "potential step" method (a), waveform for the experiment (b), and current response as a function of time (c).

However, in the initial diffusion stage, lots of positive ions move to the electrode-electrolyte interface; this also generates a large reduction current, because the difference in concentration is large. With passing time, since the difference in concentration decreases, the speed of diffusion of the positive ions decreases and, consequently, the reduction current also decreases[8]. In other words, when the current in the initial stage is measured by the "potential step" method, the characteristics of the oxidation-reduction currents are dependent on the electrostatic property of the charged molecule layer. If the molecules immobilized on the electrode can supply or accept some electrons, they can affect the current flow, especially in the initial stage after supplying the potential step.

\subsection{Experimental method and materials}

Fig. 2(a) shows a schematic diagram for the experimental setup used to analyze the current variation as a function of the antigen-antibody reaction using a potentiostat. In this system, the potentiostat is set to supply a potential-step waveform and to measure the current response from the electrochemical cell, which is filled with phosphate buffered saline(PBS) solution and consists of a disk-type platinum $(\mathrm{Pt})$ reference electrode and a circular $\operatorname{gold}(\mathrm{Au})$ electrode. The waveform has three potential steps and an amplitude of $2 \mathrm{~V}$, as shown in Fig. 2(b). The "potential step" method was performed at room temperature using a Bio-Logic SAS potentiostat / galvanostat(Bio Logic Science Instruments), controlled by EC-Lab software version 9.46 

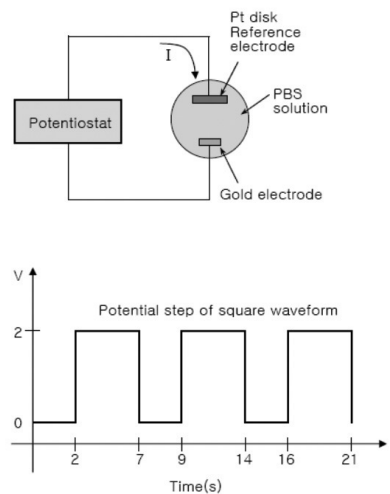

Fig. 2. Schematic diagram of potential-step experiment using potentiostat (a) and square waveform with three potential steps and amplitude of $2 \mathrm{~V}$.

PBS(pH 7.4, GIBCO) solution, Biotin PEG disulfide (Polypure), and Streptavidin(Sigma) were used in this experiment. The biotin PEG disulfide solution was prepared by dissolving it in PBS solution; its final concentration was $10 \mathrm{mM}$. The streptavidin was dissolved in PBS solution to obtain a concentration of $2 \mu \mathrm{M}$. Before the experiments, the biotin and streptavidin solutions were stored in a refrigerator at $4^{\circ} \mathrm{C}$, but the $10-\mathrm{mM}$ PBS solution was kept at room temperature and used in all rinsing processes in this experiment.

\subsection{Design and fabrication of the electrodes}

Fig. 3 shows the image of the electrodes fabricated on a glass substrate. The fabrication of the electrodes was begun by cleaning 4-inch glass wafers(Boro-33 0.5t, SCHOTT), after which a photoresist was spin-coated on them to a thickness of $1.4 \mu \mathrm{m}$ and the area of the electrodes was opened up using photolithography. Then, 200/20-nmthick $\mathrm{Au} / \mathrm{Ti}$ metal layers were evaporated and patterned by an electron beam evaporator and lift-off technique. The fabricated glass wafer was then cut into dimensions of 26 $\mathrm{mm}$ (length) $\times 16 \mathrm{~mm}$ (width). The circular gold electrodes were designed to have a diameter of $500 \mu \mathrm{m}$.

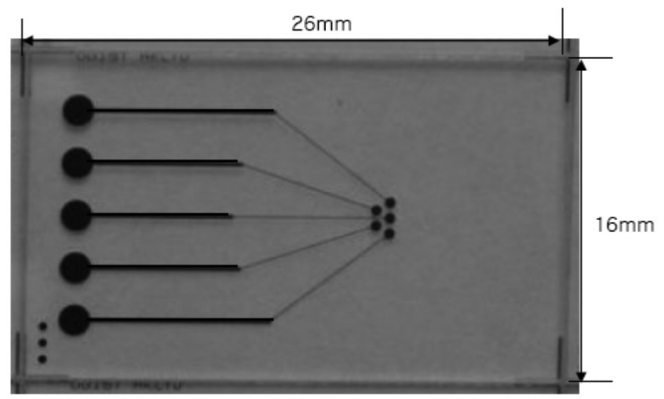

Fig. 3. The image of fabricated electrode on a glass substrate.

\subsection{Measurement of the current response}

To measure the current response of the electrochemical cell shown in Fig. 2(a), we set the glass substrate on which the gold electrode was patterned and a disk-type platinum reference electrode(2-mm diameter) in a Teflon jig and injected $500 \mu$ of $10-\mathrm{mM}$ PBS solution(pH 7.4) into the vessel. The Teflon jig was designed such that the gap between the gold electrode surface on the glass substrate and the platinum electrode surface was $3 \mathrm{~mm}$ and the vessel volume available for filling with the PBS, biotin, and streptavidin solutions was $500 \mu \mathrm{l}$. In this experiment, one gold electrode placed at the center of five electrodes was used for the purpose of achieving a simple measurement system, because its purpose was to evaluate the detection characteristics of the antigen-antibody reaction using a gold electrode by the "potential step" method. The gold and platinum electrodes assembled in the Teflon jig were connected electrically by the $10-\mathrm{mM}$ PBS solution in the vessel.

For this study, three experimental phases were designed: the first phase is used to measure the current response of the bare gold electrode, the second phase is used to measure the current response of the modified gold electrode that is immunoassayed by the biotin-PEGdisulfide, and the third phase is for the reaction of streptavidin molecules with the biotin molecules.

All measurements were repeated three times and the recorded data were averaged. The experimental results of the three phases were obtained in the following sequence. For this experiment, the gold electrode was incubated for about one hour in PBS solution to ensure the electrochemical equilibrium condition of the gold electrode. In the first phase, to record the current response of the bare gold electrode using the measurement system, the PBS solution was drained and $500 \mu \mathrm{l}$ of fresh PBS solution was introduced into the vessel. Then, the measurement system was operated three times to obtain the current response. Next, for the immobilization of the biotin PEG disulfide monolayer on the bare gold electrode, the PBS solution was drained and $500 \mu \mathrm{l}$ of a $10-\mathrm{mM}$ solution of biotin PEG disulfide in PBS was injected into the vessel. To immunoassay the biotin molecules on its surface, the gold electrode was incubated for 12 hours at room temperature. The gold electrode was rinsed five times using fresh PBS solution and, subsequently, $500 \mu \mathrm{l}$ of fresh PBS was introduced. The second experimental phase involved repeating the measurement three times. In the 
second phase, the biotin PEG disulfide molecules were grafted onto the surface of the gold electrode.

For the antigen-antibody reaction of streptavidin with the biotin PEG disulfide monolayer, $500 \mu \mathrm{l}$ of $2 \mu \mathrm{M}$ streptavidin dissolved in PBS was introduced into the vessel and incubated for 12 hours at room temperature, following the draining process of the PBS solution. The last rinsing process using fresh PBS, like the previous phase, was followed by the injection of $500 \mu$ of fresh PBS solution. The third experimental phase for the streptavidin bound to the biotin PEG disulfide layer on the gold electrode surface was then performed.

\section{RESULT AND DISCUSSION}

\subsection{Current response}

The results of the current response measurements are shown in Fig. 4. For this experiment, a potential step was supplied for five seconds after applying zero voltage for 2 s. The current response indicated that lots of positive ions, such as $\mathrm{K}+, \mathrm{Na}+$, etc., which were situated near the electrode-electrolyte interface, were reduced with passing time. This means that the positive ions received electrons from the electrode and became neutral molecules. Therefore, a very large current was generated in an instant, at 2, 9, and $16 \mathrm{sec}$ corresponding to the moments when the potential step of $2 \mathrm{~V}$ was supplied to the reference electrode. The current then decreased exponentially thereafter. In other words, when the potential was sufficient to generate a reduction current by supplying positive ions from the solution to the electrode, a large reduction current occurred across the electrode-electrolyte interface and decreased as time passed.

Fig. 4 shows that the decrease in the current induced by the immobilization of biotin PEG disulfide molecules onto the bare gold electrode was greater than that observed in the previous phase, which was performed for the bare gold electrode. Generally, the current flowing occurred across the electrode-electrolyte interface is described by electron transfer[9, 10].

Based on this result, it can be inferred that the biotin PEG disulfide molecule layer prevents the current flow. In other words, the long-chain molecules of biotin PEG disulfide hamper the electron transfer[11]. However, the result also shows that the increase in the current induced by the reaction of the streptavidin molecules with biotin was

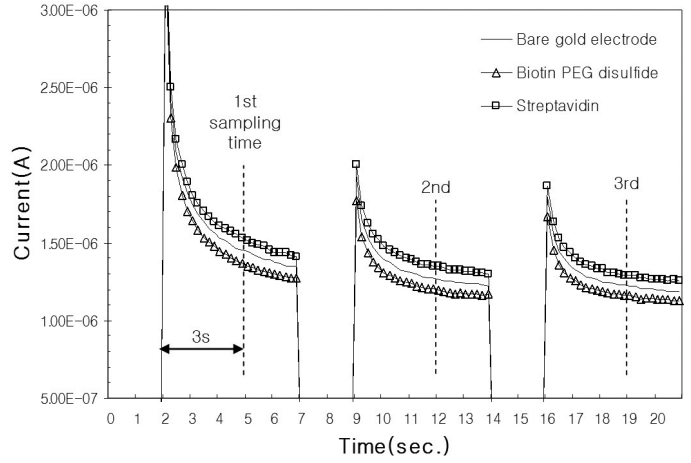

Fig. 4. Current responses of the "potential step" method for three experimental phases as a function of time.

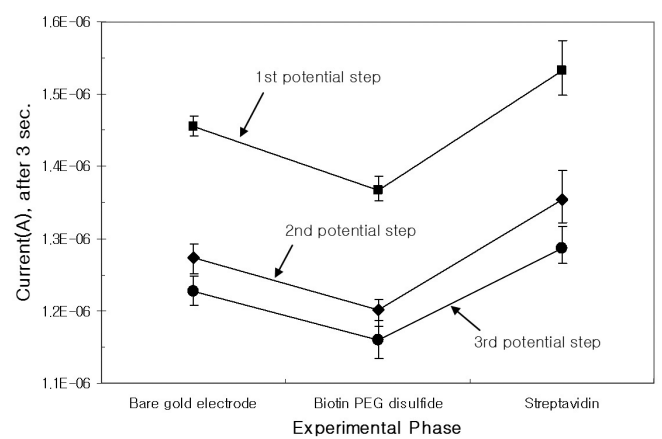

(a)

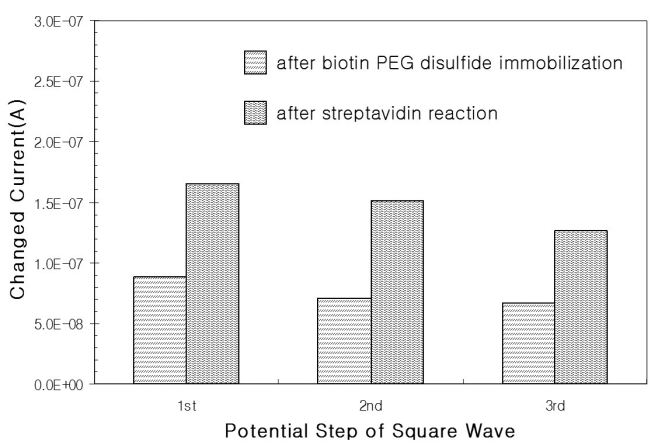

(b)

Fig. 5. Sampling data from the current response characteristics (a) and the current variation (b) as a function of the three experimental phases.

greater than that in the previous experimental phase. The electrostatic properties of streptavidin have been reported on by several previous researchers[8, 12], who indicated that the biotin binding surface of streptavidin at the molecular level formed anegatively charged molecular layer. Therefore, we suppose in this study that the streptavidin layer also forms a negatively charged molecular layer, thus creating the conditions in which more positive ions exist near the interface in the initial stage. When the voltage of the potential step was supplied, this condition allows for greater current flow than that in the 
previous experimental phase due to the deoxidization of the positive ions near the negatively charged streptavidin molecular layer.

\subsection{Data sampling}

When considering the development of a simple portable biosensor system using this "potential step" method, the problem arises as to how to record all of the data from the sensor device. To circumvent this problem, we could check the current signal at a fixed time[8]. In this experiment, a three potential step was supplied to monitor the current response and the current signal was sampled 3 seconds later.

Fig. 5 (a) shows the sampling data obtained from the current response characteristics and Fig. 5 (b) shows the variation of the response current as a function of the order of the potential step in the three experimental phases. In the order of the three potential steps, the difference in the current variation is not large, but the first potential step is larger than the others and the magnitude of the current is also the largest in this step. These results show that one potential step is sufficient to detect the variation of the current owing to the changed surface state of the electrode.

After the immobilization of biotin PEG disulfide in the first potential step, the current was $1.37 \mu \mathrm{A}$, and after the streptavidin reaction it was $1.54 \mu \mathrm{A}$. By calculating the resistance of the current flow or the resistance of electron transfer for each point, the Ret-biotin value, which is caused by the immobilization of the biotin PEG disulfide, was found to be $1.46 \mathrm{M} \Omega$, and the Ret-strep value caused by the streptavidin reaction was found to be $1.30 \mathrm{M} \Omega$. The changes in the currents caused by the immobilization of the biotin PEG disulfide molecules and the reaction of the streptavidin with the biotin were about $90 \mathrm{nA}$ and 170 $\mathrm{nA}$, respectively.

\section{CONCLUSION}

In this paper, we demonstrated that the "potential step" method using a gold electrode and a platinum reference electrode can be used to detect the reaction of streptavidin molecules with biotin. We also showed the characteristics of the current response at each "potential step" after applying a series of three potential steps with a period of seven seconds. The decrease of the current after the immobilization of the biotin PEG disulfide molecules on the bare gold electrode was greater than that for the bare gold electrode. However, the increase in the current induced by the reaction of the streptavidin molecules with the biotin is greater than that for the electrode with the immobilized biotin PEG disulfide molecules. The sampling data obtained from the experiment shows that the "potential step" method and two-electrode method are sufficient to detect the variation of the current caused by the changed surface state. This work is meaningful in that it provides a new approach for the miniaturization, integration, and low-cost fabrication of biosensor systems which can be used to detect pathogenic biomolecules. We expect that the "potential step" method can be useful to detect the event of an antigen-antibody reaction and that it can be extended to applications in which its use is combined with other devices or ICs, such as BJTs, FETs, MOSFETs and OP amps.

\section{ACKNOWLEDGMENTS}

This work was supported by the DGIST Basic Research Program of the Ministry of Education, Science and Technology(MEST) of Korea and the BK21 program.

\section{REFERENCES}

[1] C. L. Morgan, D. J. Newman, and C. P. Price, "Immunosensors : technology and opportunities in laboratory medicine", Clinical Chemistry, 42:2, pp. 193-209, 1996.

[2] M. Pedrero, S. Campuzano, and J. M. Pingarron, "Electroanalytical sensors and devices for multiplexed detection of foodborne pathogen microorganisms", Sensors, vol. 9, pp. 5503-5520, 2009.

[3] Bergveld, P., "A critical evaluation of direct electrical protein detection methods", Biosens. Bioelectron., vol. 6, no. 1, pp. 55-72, 1991.

[4] Hirst, E. R., Yuan, Y. J., Xu, W. L., and Bronlund, J. E., "Bond-rupture immunosensors-A review", Biosens. Bioelectron., vol. 23, no. 12, pp. 1759-1768, 2008.

[5] A. D’Amico, C. Di Natale, E. Martinelli, L. Sandro, and G. Baccarani, "Sensors small and numerous : always a winning strategy?", Sens. Actuators, B: Chemical, 106, (1), 29, pp. 144-152, 2005.

[6] N. Bojorge Ramirez, A. M. Salgado and B. Valdman, "The evolution and developments of immunosensors 
for health and environmental monitoring : problems and perspectives", Brazilian Journal of Chemical Engineering, vol. 26, no. 2, pp. 227-249, 2009.

[7] Allen J. Bard and Larry R. Faulkner, Electrochemical methods: fundamentals and applications 2 nd ed., John wiley \& Sons, pp. 24-28, 2001.

[8] Allen J. Bard and Larry R. Faulkner, Electrochemical methods: fundamentals and applications 2nd ed., John wiley \& Sons, pp. 156-159, 2001.

[9] J. A. Lee, S. Hwang, J. Kwak, S. I. Park, S. S. Lee, and K.-C. Lee, "An electrochemical impedance biosensor with aptamer-modified pyrolyzed carbon electrode for label-free protein detection", Sensors and Actuators B, vol. 129, pp. 372-379, 2008.

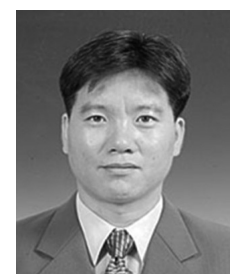

Hong-Kun Lyu received his master's degree from Kyungpook National University in 2003. Since 2005, he has been working for Daegu Gyeongbuk Institute of Science \& Technology in Republic of Korea. His current research is focused on semiconductor devices and applications.

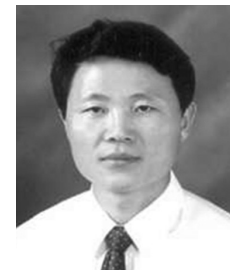

Yoon Soo Han received his Ph.D. in Polymer Science and Engineering from Kyungpook National University, Korea in 2000. From Jan. 2005 to Feb. 2011, he worked as a Principal Researcher in Daegu Gyeongbuk Institute of Science $\&$ Technology (DGIST). Since Mar. 2011, he has been a professor in Department of Advanced Energy Material Science and Engineering, Catholic University of Daegu. His research interests are electronic materials and devices such as solar cells, displays and sensors.
[10] Alan K.H. Cheng, Dipankar Sen and Hua-Zhong Yu, "Design and testing of aptamer-based electrochemical biosensors for proteins and small molecules", Bioelectrochemistry, vol. 77, Issue 1, pp. 1-12, 2009.

[11] S.-J. Ding, B.-W. Chang, C.-C. Wu, M.-F. Lai, and H.-C. Chang, "Electrochemical evaluation of avidin biotin interaction on self-assembled gold electrodes", Electrochimica acta, vol. 50, Issue 18, pp. 3660-3666, 2005.

[12] S. Sivasankar, S. Subramaniam, and D. Leckband, “ Direct moleculear level measureemts of the electrostatic properties of a protein surface", Proc. Natl. Acad. Sci. USA, vol. 95, pp. 12961-12966, USA, October 1998.

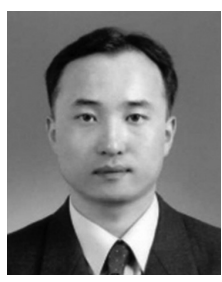

Sungho Woo received his M.Sc. degree from POSTECH in 1997. Since 2005, he has been working for Daegu Gyeongbuk Institute of Science \& Technology (DGIST) in Republic of Korea. His current research is focused on the development of novel hybrid materials and device structures for applications in optoelectronic devices, such as OLEDs and solar cells.

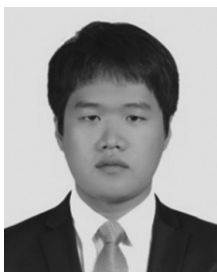

Hee-Ho Lee received his bachelor ${ }^{\circ}$ s degree from Kyungpook National University in 2009. He is currently working toward the master ${ }^{\circ} \emptyset$ s degree in the School of Electronics Engineering, college of IT engineering of Kyungpook National University.

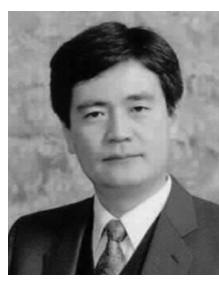

Jang-Kyoo Shin received his Ph.D. in Dept. of Electrical and Computer Engineering, Colorado State University, Fort Collins, Colorado, USA. He is a professor in the School of Electronics Engineering, college of IT engineering of Kyungpook National University, from 1980 up to now. Since 2010, He has been working as president of Institute of Semiconductor Fusion Technology in the campus. His research interests include semiconductor devices and sensors such as biosensors and image sensors. 